\title{
Design Techniques and Paradigms Toward Design of Low-Voltage CMOS Analog Circuits
}

\author{
Vladimir I. Prodanov \& Michael M. Green \\ University at Stony Brook \\ Stony Brook, NY 11794-2350 \\ mgreen@ee. sunysb.edu, vip@ee.sunysb.edu
}

\begin{abstract}
Techniques for the design of robust low-voltage CMOS analog circuits are given. It is shown that both $V_{t^{-}}$ independence and proper alignment between the input and output swings are crucial for effective design of low-voltage circuits.
\end{abstract}

\section{INTRODUCTION}

Virtually everyone involved in the design of integrated circuits is aware of the strong push to operate at everdecreasing supply voltages. Many IC companies are manufacturing circuits that operate at $3.3 \mathrm{~V}$ for low-voltage/lowpower operation such as laptop computers. Now many companies are looking toward operation at even lower supply voltages, even down to $1 \mathrm{~V}$, in the not-too-distant future.

Given that in a standard CMOS fabrication process most of the important parameters for design (e.g., threshold voltage) will be optimized for operation of digital circuits, without a doubt the possibility of robust analog circuits working down to $1 \mathrm{~V}$ will require a major paradigm shift in the way we think about design. In this paper we will discuss some ideas for designs that can accommodate operation near $V_{D D}=1 \mathrm{~V}$ without requiring any special process considerations. In particular, we make the following assumptions:

1. No special fabrication steps are required; in particular, all threshold voltages are dictated by considerations related to the digital circuitry.

2. All MOS transistors operate in strong inversion to allow high speed design (e.g., video/radio frequencies).

Low-voltage BJT circuits are already quite common. Designing CMOS analog circuits operating at similar low supply voltages is far more difficult, however, due to some fundamental differences in the behavior of these devices. These differences are illustrated in Table I. In this table, $V_{T}$ denotes the thermal voltage $k T / q ; V_{t}$ denotes the MOS threshold voltage; $V_{A}$ denotes the BJT Early voltage; $W_{B}$ denotes the BJT base width; $\Delta V_{G S} \equiv V_{G S}-V_{t}$ for an MOS transistor. Notice that the intrinsic gain and unity-gain frequency are, in a first-order analysis, dependent only on processing parameters and fundamental physical constants for the BJT, not on the transistor biasing. Since the minimum supply voltage for a BJT circuit is determined only by $V_{B E(o n)}$, obtaining low-voltage operation of BJT cir-
TABLE I

COMPARISON OF BJT AND MOS PERFORMANCE PARAMETERS.

\begin{tabular}{|l||l|l|}
\hline \multicolumn{1}{|c||}{ Parameter } & \multicolumn{1}{c|}{ BJT } & \multicolumn{1}{c|}{ MOS } \\
\hline \hline Intrinsic gain $\left(g_{m} r_{o}\right):$ & $V_{A} / V_{T}$ & $2 \lambda^{-1} / \Delta V_{G S}$ \\
\hline Unity gain frequency $\left(\omega_{T}\right):$ & $2 \frac{\mu}{W_{B}^{2}} \cdot V_{T}$ & $\frac{3}{2} \frac{\mu}{L^{2}} \cdot \Delta V_{G S}$ \\
\hline \hline Minimum supply voltage: & $V_{B E(o n)}$ & $V_{t}+\Delta V_{G S}$ \\
\hline Bias current $\times 10$ results in: & $V_{B E}+60 \mathrm{mV}$ & $\Delta V_{G S} \times 3.2$ \\
\hline
\end{tabular}

cuits while retaining sufficient performance parameters is straightforward. The dependence of these parameters on $V_{T}$ for the BJT case, however, is replaced by $\Delta V_{G S}$ for the MOS case, which is of course bias dependent. The minimum supply voltage will be determined by $V_{t}+\Delta V_{G S}$, which is also bias dependent. Moreover, threshold voltages are unlikely to drop below $0.4 \sim 0.5 \mathrm{~V}$ even as MOS feature sizes continue to decrease due to constraints from the digital circuitry. Thus there exist significant obstacles to the design of low-voltage, high-performance analog CMOS circuits. These obstacles are exacerbated by the fact that $\Delta V_{G S}$ is much more sensitive to increases in bias current than $V_{B E}$, also illustrated in Table I. This fact impacts the low-voltage design of circuits that require adjustable bias currents, such as tunable continuous-time filters.

To illustrate the difficulty of obtaining low-voltage operation of a CMOS circuit, consider the standard CMOS folded-cascode amplifier shown in Fig. 1(a). The output range $\Delta V_{\text {out }}$ of this circuit is determined by the values of $V_{\text {out }}$ for which transistors $M_{7}$ and $M_{9}$ stay in the saturation region. In order to simplify this analysis, we assume that the threshold voltage and $\Delta V_{G S}$ are the same for all transistors. In this case, as illustrated by the chart shown in Fig. 1(a), $V_{\text {out }}$ can swing within $V_{t}+2 \Delta V_{G S}$ of either rail. Likewise, the input common-mode swing $\Delta V_{C M}$ is determined by the values of $V_{C M}$ for which transistors $M_{1}$, $M_{2}$ and $M_{3}$ stay in the saturation region. This range is also illustrated in the Fig. 1(a) chart. If we now assume that this amplifier is to be used in a unity-gain configuration (this is the most stringent condition with regard to dynamic range), then the overall swing $\Delta V \equiv \Delta V_{\text {out }} \cap \Delta V_{C M}$ which in this case is $\Delta V_{o u t}=V_{D D}-\left(2 V_{t}+4 \Delta V_{G S}\right)$. Clearly this circuit is not of much use for $V_{D D}<5 \mathrm{~V}$. 

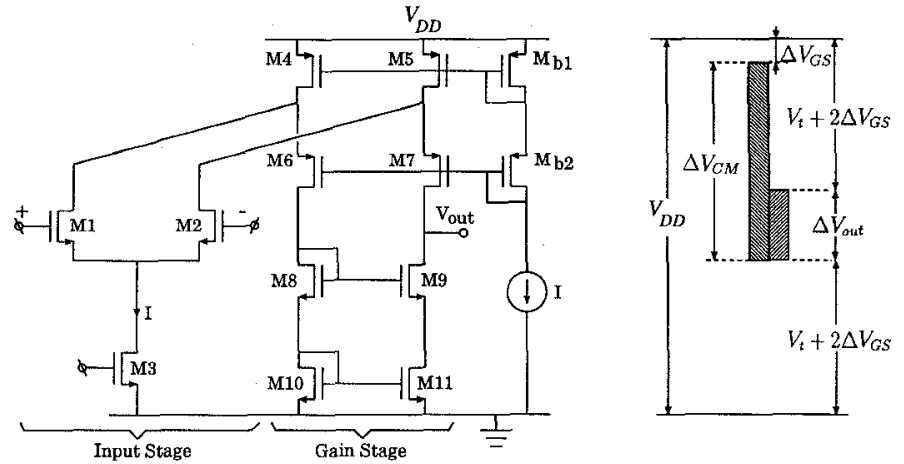

(a)
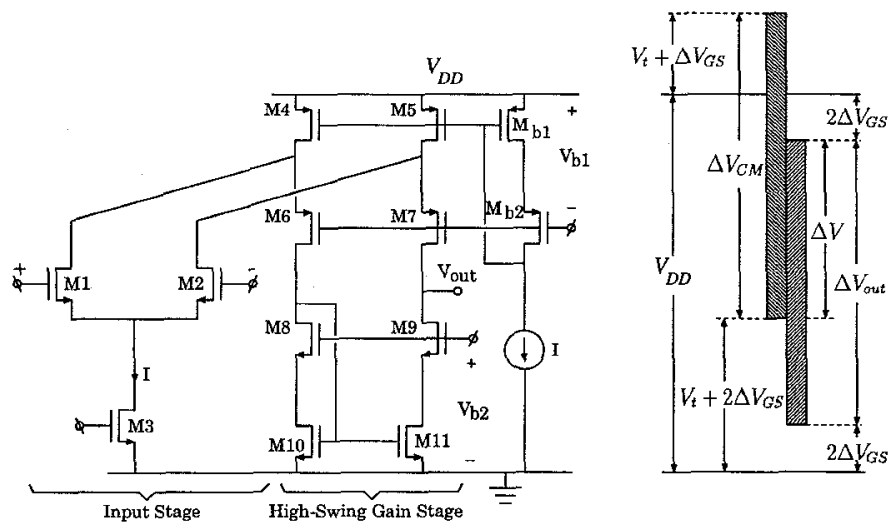

(b)

Fig. 1. (a) Standard folded-cascode CMOS amplifier; (b) High-swing folded-cascode amplifier.

A well known method for increasing the swing of the folded-cascode amplifier is shown in Fig. 1(b). In this "high-swing" amplifier, the transistors are biased such that $V_{\text {out }}$ can swing $V_{t}$ closer to either rail, as illustrated in the chart shown in Fig. $1(\mathrm{~b})$; i.e., $\Delta V_{\text {out }}=V_{D D}-4 \Delta V_{G S}$. Notice that this expression for $\Delta V_{\text {out }}$ does not involve $V_{t}$. This property, called " $V_{t}$-independence," is very desirable for low-voltage operation. Using a similar analysis as before, the input common-mode swing $V_{C M}=$ $V_{D D}-\left(V_{t}+2 \Delta V_{G S}\right)$.

Although this structure can operate at lower supply voltages than the standard folded cascode amplifier, it has several disadvantages:

1. The $V_{t}$-independence of the output swing $\Delta V_{\text {out }}$ is based upon several transistors being biased on the edge of the their" saturation region. In order to make the circuit more robust, there is usually a margin of $100 \sim$ $200 \mathrm{mV}$ designed into the circuit. This margin detracts directly from the output swing.

2. The input stage is $V_{t}$-dependent, thereby making the overall swing $V_{t}$-dependent.

3. The supply voltage must be at least $2 V_{t}$ in order to properly bias all transistors.

The following section addresses these disadvantages with some novel circuit topologies.

\section{Design of Novel Low-Voltage Analog Blocks}

\section{A. Low-Voltage Current Mirrors}

One can show that the voltage-current relation of the stacked-transistor configuration shown in Fig. 2, where $M$ is biased in triode, is given by:

$$
I_{D}+\left(1+\frac{\beta}{\beta_{b}}\right) I_{b}=\frac{\beta}{2}\left(V_{G S}-V_{t}\right)^{2}
$$

Hence, the stacked-transistor topology with input current $I_{D}$ has the same $V_{G S}$ as that of a diode-connected MOS transistor conducting current $I_{D}+\left(1+\frac{\beta}{\beta_{b}}\right) I_{b}$. This idea can be used to configure linear current mirrors with reduced input and/or output voltage requirements (e.g. Fig. 3). The drawback of the Fig. 3(a) and Fig. 3(b) low-voltage current mirrors is the presence of an offset. An offset-free version of a low-voltage Wilson current mirror is shown in Fig. 3(c). It has $V_{t}$-independent input and output voltage requirements and output resistance on the order of $g_{m} r_{o}^{2}$. The constraint on the supply voltage for this circuit is given by:

$$
V_{D D} \geq V_{t}+2 \Delta V_{G S}+V_{D S 1,2}
$$

Simulations show that the circuit behaves as a current mirror with $r_{\text {out }}>10 \mathrm{M} \Omega$ for $V_{\text {in }} \geq 200 \mathrm{mV}, V_{\text {out }} \geq 250 \mathrm{mV}$ and $V_{D D} \geq 1.2 \mathrm{~V}$ with $V_{t}=0.9 \mathrm{~V}$. More details on this circuit are given in [1].

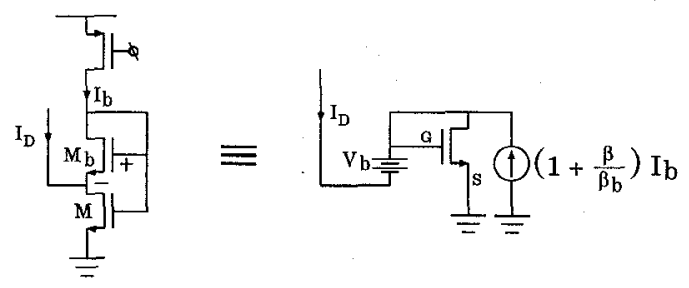

Fig. 2. Stacked-transistor topology and its equivalent.

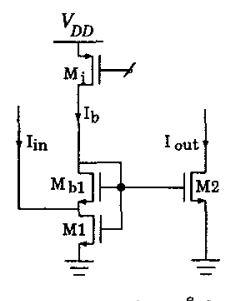

$I_{\text {out }}=I_{\text {in }}+\left(1+\frac{\beta}{\beta_{b}}\right) I_{b}$ (a)

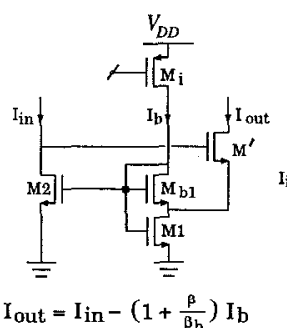

(b)

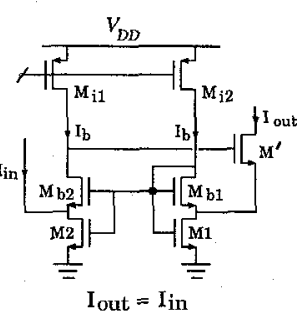

(c)
Fig. 3. Low-voltage current mirrors: (a) simple topology; (b) Wilson topology; (c) Offset-free Wilson-like topology; 

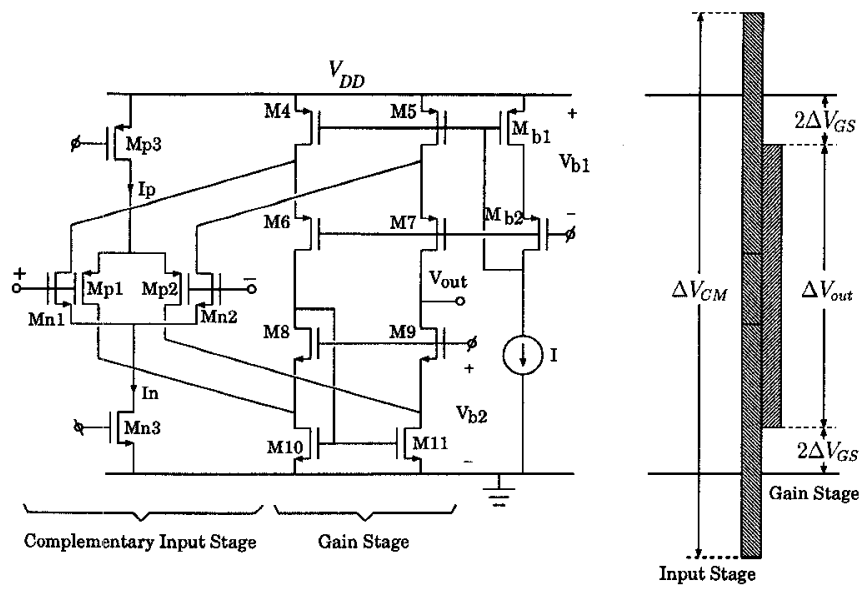

Fig. 4. Rail-to-rail op-amp using complementary input stage

\section{B. Constant $-g_{m}$ Complementary Input Stage}

The underlying reason for the $V_{t}$-dependence of the swing of the Fig. 1(b) amplifier is the fact that for sufficient common-mode rejection, a current source is required to bias the input differential pair. It is this transistor that limits the common-swing toward the negative rail. To achieve rail-to-rail input swing the use of complementary input stage, with an n-channel and p-channel differential pair driven in parallel (e.g. Fig. 4) has been proposed. If the tail currents are kept constant then this input stage will have a transconductance that varies with the common-mode input, which is very undesirable in an amplifier. However, if all transistors are biased in strong inversion and a constant transconductance is desired, then the relation

$$
\sqrt{\beta_{n} I_{n}}+\sqrt{\beta_{p} I_{p}}=\text { constant }
$$

must be satisfied. Circuits realizing (2) have been reported [2]-[5]. However, these circuits tend to be quite complex and most assume tracking of the $k^{\prime}$ between $\mathrm{n}$-channel and p-channel transistors [3]-[5], which is undesirable for robust operation over processing variations.

A better approach is based upon using the two-stage structure shown in Fig 5. It can be shown, assuming the p-channel transistors are all of identical dimensions, that

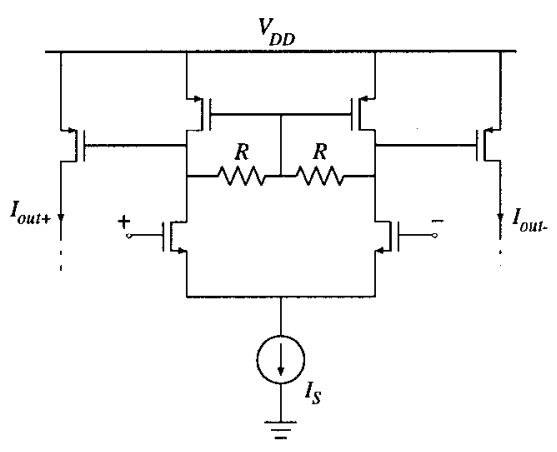

Fig. 5. Two-stage differential pair

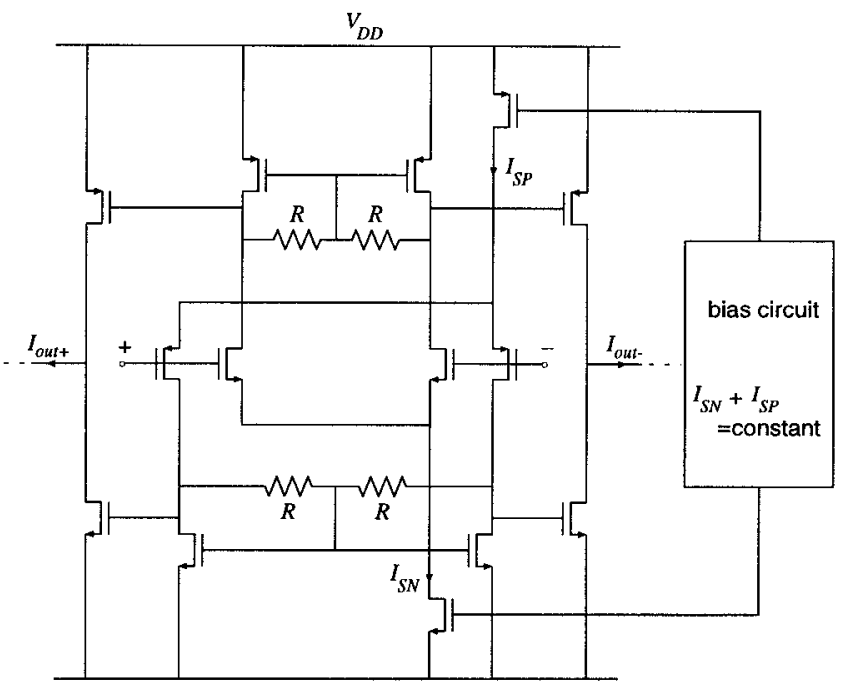

Fig. 6. Complementary input stage using two-stage differential pairs.

the effective transconductance of this block is given by:

$$
g_{m(\text { eff })}=\frac{i_{\text {out }+}-i_{\text {out- }}}{v_{+}-v_{-}}=\sqrt{\beta_{n} \beta_{p}} R I_{S}
$$

If this two-stage differential pair is used in a complementary fashion as shown in Fig. 6, then the overall effective transconductance is given by:

$$
g_{m(e f f)}=\sqrt{\beta_{n} \beta_{p}} R\left(I_{S N}+I_{S P}\right)
$$

In order to keep $g_{m(e f f)}$ constant, all that is needed to keep the sum of two dc currents constant, which requires only simple circuitry [7], [8]. Notice that no matching of $\mathrm{n}$-channel and p-channel parameters is required.

Notice that the Fig. 5 two-stage input stage has a transconductance that is proportional to the bias current itself, rather than proportional to the square root of the bias current, as with conventional MOS strong-inversion differential pairs. Hence this block is also useful for wide-range tunable applications.

\section{Input/Output Swing Alignment Using On-Chip Charge Pump}

Although the complementary input stage discussed above is an improvement over previous reported results, there are two problems associated with any kind of complementary input stage. First, a complementary input stage does extend the common-mode swing however the biasing of both differential pairs still constrains $V_{D D}>$ $2 V_{t}+2 \Delta V_{G S}$. Second, the nonlinear behavior inherent in such an input stage (i.e., switching between differential pairs) makes it very difficult to accommodate linear operation with a large differential input (e.g., a linear $V-I$ converter). Attempts to design such circuits results in a high amount of complexity [9]. To overcome both of these obstacles, the following technique can be used.

First we observe from Fig. 1(b) that $\Delta V_{C M} \approx \Delta V_{\text {out }}$. Hence, the limitation on input/output swing is in fact not 
due to the individual input/output swings, rather the relative alignment of these two ranges. If it could be arranged that the common-mode range is simply shifted down by $V_{t}$, then the two ranges would be aligned, thus giving a much higher overall swing. A technique for shifting the commonmode range is shown in Fig. 7. (The high-swing gain stage is not shown in the Figure.) The input range is shifted down by $V_{t}+\Delta V_{G S}$ by the source-follower transistors $M_{2}$. However, in order to keep current sources transistors $M_{3}$ properly biased for common-mode ranges near the positive rail, their sources must be higher than $V_{D D}$. Assuming that an on-chip clock is available from the digital circuitry, such a voltage can be generated using an on-chip charge pump [10]. Notice that this charge pump is only required to provide current $2 I_{\text {shift }}$ which would typically be quite small. Hence, this charge pump, including the filtering capacitor, could be built entirely on the chip without the requirement of extra pins. Note that since this input stage is based on a simple differential pair, standard linearization techniques, such as source degeneration, can be used.
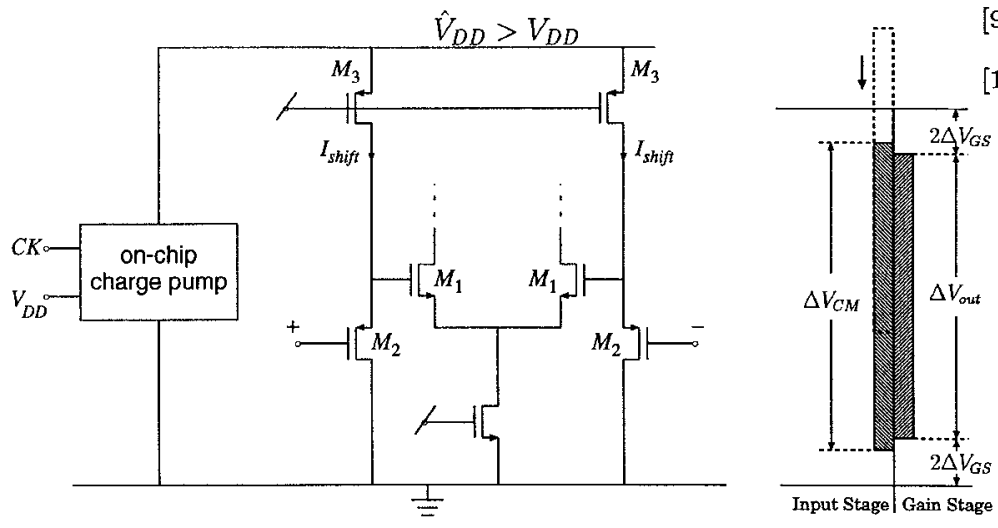

Fig. 7. Differential pair with on-chip charge pump to align its swing with that of the gain stage

\section{Conclusion}

A number of techniques for design of CMOS analog circuits for low-voltage applications have been presented. It was shown that $V_{t}$-independence and input/output swing alignment are powerful techniques for designing robust lowvoltage analog circuits.

\section{REFERENCES}

[1] V. I. Prodanov and M. M. Green, "CMOS current mirrors with reduced input and output voltage requirements," Electronics Letters, v. 32, 18 Jan. 1996, pp. 104-105.

[2] S. Sakurai and M. Ismail, LOW-VOLTAGE CMOS OPERATIONAL AMPLIFIERS: Theory, Design and Implementation. Kluwer Academic Publishers, 1995.

[3] H. Huijsing, R. Hogervorst and K. de Langen, "Low-power lowvoltage VLSI operational amplifier cells," IEEE Transactions on Circuits and Systems - Part I, vol. 42, pp.841-852, Nov. 1995.

[4] K. Nagaraj, "Constant transconductance CMOS amplifier input stage with rail-to-rail input common mode voltage," IEEE Transactions on Circuits and Systems - Part II, vol.42, pp.366-368, 1995.

[5] A. L. Coban and P. E. Allen "A low-voltage CMOS op amp with rail-to-rail constant- $g_{m}$ input stage and high-gain output stage," Proc. of ISCAS, vol.2, pp. 1548-1551, 1995.

[6] C. Hwang, A. Motamed and M. Ismail, "Universal constant- $g_{m}$ input-stage architectures for low-voltage op amps," IEEE Transactions on Circuits and Systems - Part I, vol. 42, pp. 886-894, Nov. 1995.

[7] H. Huijsing and D. Linebarger, "Low-voltage operational amplifier with rail-to-rail input and output ranges,"Journal of SolidState Circuits, vol. sc-20, pp.1144-1150, Dec. 1985.

[8] J. H. Botma, R. F. Wassenaar, R. J. Wiegerink, "Simple rail-torail low-voltage constant-transconductance CMOS input stage in weak inversion," Electronics Letters, vol. 29, No. 12, pp. 11451147, June 1993.

[9] P. P. Vervoort and R. F. Wassenaar "A CNOS rail-to-rail linear V-I converter," Proc. of ISCAS, vol.2, pp. 825-828, 1995.

[10] J. F. Dickson, "On-chip high-voltage generation in NMOS integrated circuits using an improved voltage multiplier technique," Journal of Solid-State Circuits, vol. 11, pp. 374-378, June 1976. 